\title{
COMMISSION 19: ROTATION OF THE EARTH
}

\author{
(ROTATION DE LA TERRE)
}

PRESIDENT: Nicole Capitaine

VICE-PRESIDENT: Véronique Dehant

ORGANIZING COMMITTEE: G. Beutler, P. Brosche, A. Brzezinski, T. Fukushima, D. Gambis, R. Gross, J. Hefty, C. Huang, Z. Malkin, D. McCarthy, A. Poma, J. Ray, B. Richter, C. Ron, N. Sidorenkov, M. Soffel, C. Wilson, Ya.Yatskiv

\section{INTRODUCTION}

The commission held business and scientific sessions at the General Assembly on Friday 18 July 2003. One scientific session has been devoted to the Reports from Services (IERS and IVS) and the Working Group on "Precession-nutation", of which complete reports can be found in the IAU Transactions (Vol XXVA, 2003, H. Rickmann ed.). The other scientific session was devoted to (i) IERS2000 Conventions: comparisons of models with observations, (ii) Combination: results and prospects, (iii) The Role of the spatial missions for the determination of the Earth's gravitational potential and relation with Earth's rotation.

Regarding Services that are currently reporting to Commission 19, J. Vondrak, President of the IERS Directing Board, mentioned that the recent IERS Retreat (April 2003) led to the change of the name of the IERS (International Earth rotation and Reference frames Service) and to starting a new Combination Pilot Project.

Regarding Commission 19 Working Groups, V. Dehant, Chair of the Working Group "Precession-nutation", reported on the ITRS motion of the CIP corresponding to sub-diurnal nutations that have been recently examined by a sub-group of the WG chaired by A. Brzezinski. This group adopted a Table for operational use which has been provided in the IERS Conventions 2003. N. Capitaine gave a summary of the IAU Working Groups discussion that has been held on the equinox offset issue and explained how this offset has been taken into account in the actual implementation of the IAU 2000 precession-nutation.

\section{SCIENTIFIC PRESENTATIONS}

\subsection{Expressions to implement the IAU 2000 definition of UT1 (Capitaine, Wallace, and McCarthy)}

The implementation of the IAU 2000 resolutions, beginning on 1 January 2003, requires the adoption of a new definition of UT1 together with the adoption of the IAU 2000 model for the motion of the Celestial Intermediate Pole (CIP) in the Geocentric Celestial Reference System. This defines UT1 as linearly proportional to the Earth Rotation Angle (ERA) through a defining relation. We have provided expressions to be used to implement the IAU 2000 definition of UT1, using the IAU 2000 precession-nutation model, either in the new (CEO-based) or classical (equinox-based) transformations between the ITRS and GCRS. The new expression provided for GST is in agreement at 
the micro-arcsecond level, for one century, with the IAU 2000 expressions for the ERA and for the quantity s. The computations of the new expressions are performed in such a manner as to ensure that there is no discontinuity in UT1 on 1 January 2003 and that there is equivalence of the classical and new transformations between the ITRS and GCRS relative to the rotation about the axis of the CIP when these expressions are used.

\subsection{ERA-UT1 and GAST-UT1 Relations (Fukushima)}

Based on the latest precession theory (Fukushima 2003), we derived two quantities locating CEO in the pre-2003 and post-2003 IAU formulations. Then we obtained the relations between UT1 and GAST in the pre-2003 formulation, and between UT1 and Earth Rotation Angle (ERA) in the post-2003 one. Both of them lead to the same $\mathrm{CEO}$ on the true equator. The periodic parts in the relations are almost the same as given in Capitaine et al. (2003). While the secular part does differ from Capitaine et al. (2003) and Bretagnon et al. (2003). These differences come from those in the adopted precession formulae.

\subsection{Tests of the ITRS to GCRS Transformation based on VLBI data (Gon- tier)}

The analysis of an Earth based observation of a celestial object requires an astrometric model to express the transformation from a geocentric terrestrial frame to a geocentric celestial one. The classical transformation refers to the equinox of date and uses classical precession and nutation quantities. In a new astronomical modeling approach we refer to the non-rotating-origin on the equator of date, proposed by Guinot (1979) and use the celestial coordinate of the CIP (Capitaine, 1990). Resolution B1.8 adopted by the IAU XXIV General Assembly, recommends the use of this new approach. At first, we present the implementation of the IAU 2000 resolutions in the VLBI analysis software "GLORIA". Then we show various tests performed on VLBI observations in order to verify the level of agreement between the two transformations.

\subsection{IAU2000 Nutation model versus combined VLBI/GPS solutions (Von- drák)}

The International Astronomical Union adopted in 2000 the new model of precessionnutation, based on Souchay et al. (1999) solution for the rigid Earth and the transfer function by Mathews et al. (2002) based on a complex geophysical Earth model. Some parameters of the model were estimated by a fit to VLBI observations made in 19791999 at weekly intervals. Now the International VLBI Service provides a combined solution with a higher time resolution, and the European center of International GPS Service provides a solution of daily nutation rates, derived from GPS observations. These data suffer from long-periodic systematic errors, most probably due to GPS orbit mis-modeling. A procedure is proposed to constrain the long-periodic part of GPS-based nutation rates to VLBI, and then to combine both series to obtain highresolution solution of precession-nutation. The combined VLBI/GPS solution in the interval 1994.3-2003.0 is compared with the new model and discussed. The combination of VLBI with GPS observations provides a higher resolution and a better fit to the new model than the VLBI observations alone; it is on the level of $0.10-0.15$ mas, if longperiodic part (FCN) is removed. 


\subsection{IERS EOP/PC Results in the new IAU System (Gambis and Bizouard)}

Beginning on 1 January 2003, a new combined solution of $\mathrm{dX}$ and $\mathrm{dY}$ related to the new IAU 2002A precession-nutation theory in addition to the current $(\mathrm{d} \psi, \mathrm{d} \epsilon)_{1980}$. The analyses of these temporal series are performed. Possibility of estimating the free core $(\mathrm{FCN})$ and free inner core (FICN) signals are discussed.

\subsection{The Free Core Nutation (McCarthy)}

The recently adopted IAU 2000 precession-nutation model does not contain a model for the free core nutation. This is a free motion of the pole in space that cannot be predicted with certainty and must be observed. The International Earth rotation and Reference system Service (IERS) provides expressions that have been fit to past observations to be used to model the phenomenon. These expressions vary with time and are updated as appropriate by the IERS.

\subsection{Relation of the gravity field missions with Earth's rotation (Schuh and Fernandez)}

The present satellite missions CHAMP and GRACE and the future GOCE satellite will allow to determine the gravity field of the Earth with high spatial and temporal resolution. A short overview about the present state of these missions are given. As these satellites will play an important role for the new IAG lead project IGGOS (Integrated Global Geodetic Observing System) the relation between geometry and rotation of the Earth and it's gravity field are described with respect to the interactions of the various components of system Earth. Mass redistribution, changes of the geometry and variations of the density are common causes for variations of Earth rotation and of the gravity field. Mass redistribution in surface fluid envelopes, e.g. in the oceans and in continental water reservoirs (soil moisture, snow cover, and ground-water), in the cryosphere, and to a small amount in the atmosphere could cause such variations. The continental water storage change was studied using a model based on assimilated soil moisture and snow accumulation data from the NCEP/NCAR reanalysis. Using the trace of the Earth inertia tensor as well as C20 changes, the variations on the ERP due to this continental hydrology model are shown.

\section{BUSINESS SESSION}

Commission 19 elections for new officers, new members and consultants, that have been made electronically before the GA, have been approved. The new OC has thus been formed and new members of the Commission have been accepted.

A discussion on the future of the Commission and Commission Working Groups within the future revised by-laws of the IAU has led to a proposal of the enlargement of the scope of the Commission to reference systems. According to such an evolution, the name of the Commission would become "Earth rotation and related reference systems" and the terms of reference would include the corresponding tasks of the Division I Working Group on "the International Celestial Reference System".

Acknowledgments. I am grateful to Commission 19 Organizing Committee for the suggestions regarding the preparation of the scientific programme of the meeting and to all the contributors to Commission 19 meeting for their valuable input to the work of the Commission during the General Assembly.

Nicole Capitaine

President of the Commission 